\title{
Relaxed Utility Maximization in Complete Markets
}

\author{
Sara Biagini * Paolo Guasoni ${ }^{\dagger \ddagger}$
}

May 15, 2009

\begin{abstract}
For a relaxed investor - one whose relative risk aversion vanishes as wealth becomes large the utility maximization problem may not have a solution in the classical sense of an optimal payoff represented by a random variable. This nonexistence puzzle was discovered by Kramkov and Schachermayer (1999), who introduced the reasonable asymptotic elasticity condition to exclude such situations.

Utility maximization becomes well-posed again representing payoffs as measures on the sample space, including those allocations singular with respect to the physical probability. The expected utility of such allocations is understood as the maximal utility of its approximations with classical payoffs - the relaxed expected utility.

This paper decomposes relaxed expected utility into its classical and singular parts, represents the singular part in integral form, and proves the existence of optimal solutions for the utility maximization problem, without conditions on the asymptotic elasticity. Key to this result is the Polish space structure assumed on the sample space.
\end{abstract}

Key Words: Utility Maximization, Asymptotic Elasticity, Integral Representation

Mathematics Subject Classification (2010): 91G10, 91B16, 49J45

JEL Classification: G11

\section{Introduction}

The problem of maximizing expected utility from a set of payoffs of price $x$ :

$$
\max _{p(X)=x} E_{P}[U(X)]
$$

is central for asset pricing and portfolio choice. If the market is complete (i.e. $p(X)=E_{Q}[X]$ for some pricing measure $Q$ ), the typical solution starts from the Euler equation:

$$
U^{\prime}(X)=y \frac{d Q}{d P}
$$

which aligns marginal utility with the state price density $d Q / d P$. If the Lagrange multiplier $y$ satisfies the saturation condition $E_{Q}[X]=x$, then the payoff $X^{*}(y)=\left(U^{\prime}\right)^{-1}(y d Q / d P)$ is optimal for the problem (UM).

\footnotetext{
*Dipartimento di Statistica e Matematica Applicata all‘Economia, Università di Pisa, Via Ridolfi, 10, Pisa, Italy, sara.biagini@ec.unipi.it

${ }^{\dagger}$ Department of Mathematics and Statistics, Boston University, 111 Cummington St, Boston, MA 02215, United States, guasoni@bu.edu

${ }^{\ddagger}$ Supported by the National Science Foundation under grants DMS-0532390 and DMS-0807994
} 
This argument is so common that passing from a solution of (1) to a solution of (UM) is considered almost automatic. Checking the condition $E_{Q}[X]=x$ seems a formality, to be skipped if the actual value of $y$ is not required.

Yet, the argument may fail. For certain combinations of the utility function $U$, the state price density $Q$, and the initial capital $x$, none of the payoffs $X^{*}(y)$ satisfies $E_{Q}\left[X^{*}(y)\right]=x$, and the problem (UM) has no solution - a phenomenon first discovered by Kramkov and Schachermayer (1999, Example 5.2). Indeed, they show the existence of a solution under the asymptotic elasticity condition:

$$
A E(U)=\limsup _{x \uparrow \infty} \frac{x U^{\prime}(x)}{U(x)}<1
$$

This condition has a clear interpretation in terms of asymptotic relative risk aversion:

$$
\operatorname{ARRA}(U)=\lim _{x \uparrow \infty}-\frac{x U^{\prime \prime}(x)}{U^{\prime}(x)}
$$

When this limit exists, De l'Hôpital's rule implies that the condition $A E(U)<1$ is equivalent to $A R R A(U)>0$, i.e. relative risk aversion is bounded away from zero for arbitrarily large wealth levels. Thus, optimal payoffs may not exist for utility functions which are asymptotically relatively risk neutral, i.e. $A R R A(U)=0$.

It is tempting to dismiss such examples as mathematical pathologies without economic substance. Indeed, all common examples of utility functions, including the logarithmic, power, exponential, and in general the HARA (Hyperbolic Absolute Risk Aversion) class, do satisfy $A R R A(U)>$ 0 . This class of utility functions is ubiquitous in Finance, and a condition violated by this class seems of little interest.

However, power utilities themselves leads to a utility function satisfying $A R R A(U)=0$ in heterogeneous preferences equilibria. In a model with several agents with individual constant relative risk aversion (i.e. power utility), Benninga and Mayshar (2000) and Cvitanic and Malamud (2008) show that the utility function of the representative agent has decreasing relative risk aversion, which converges - for large levels of wealth - to the value of the least risk averse agent. Thus, the presence of agents with arbitrarily low relative risk aversion implies that $A R R A(U)=0$.

This paper studies the utility maximization problems for complete markets, relaxing the assumption $A E(U)<1$. The central idea is that the topological structure on the sample space $\Omega$ allows to obtain a solution even in the critical case $A E(U)=1$. In all models of interest, the sample space $\Omega$ is already endowed with such a topology, but the classical theory of utility maximization discards topological information, focusing on the measurable structure alone.

This loss of information is inconsequential if $A E(U)<1$ : then a random variable $X$ that maximizes expected utility always exists. But if $A E(U)=1$ and the initial $x$ capital exceeds some critical value $x^{*}$, then the agent may achieve maximal utility by concentrating capital on events of arbitrarily small probability. Thus, the candidate optimum would allocate finite capital on a set of probability zero. Alas, expected utility neglects null sets, and cannot account for such singular allocation.

The topology on $\Omega$ resolves this problem by identifying available payoffs with Radon measures $\mu$ of mass less than or equal to $x$ - the space of relaxed payoffs. Then the contribution to expected utility of $\mu$ splits into two parts. The classical expected utility $E_{P}[U(X)]$ accounts for the component $\mu_{a}=X d Q$, absolutely continuous with respect to $Q$. The component $\mu_{s}$, singular with respect to $Q$ leads to the novel term $\int \varphi d \mu_{s}$, which credits the concentration of capital on null sets for its contribution to expected utility. The "singular utility" $\varphi$ depends on both the utility function $U$ and on the pricing measure $Q$. 
This paper contributes to Mathematical Finance by resolving the nonexistence puzzle of Kramkov and Schachermayer (1999) in complete markets, proving the existence of a solution in a larger space of payoffs, and it clarifies the structure of the expected utility and its maximizers.

Mathematically, the main result is Theorem 1.3, which can be read as an integral representation result for the utility functional. In comparison with similar results in the literature, applications to Mathematical Finance require more flexible assumptions on the sample space $\Omega$, assumed to be Polish, not necessarily locally compact.

The rest of the paper is organized as follows: Section 1 summarizes the assumptions and the main results, discussing their significance. Section 2 proves the integral representation result, and it is probably the most technical part of the paper. The utility maximization result is proved in Section 3, while the last Section contains examples and counterexamples which show the relevance of the results and their assumptions.

\section{Summary of Results}

The paper makes the following model assumptions:

Assumption 1.1. Let $(\Omega, \mathcal{T})$ be a Polish space, and $P$ a Borel-regular probability on the Borel $\sigma$-field $\mathcal{F}$.

i) The utility function $U:(0,+\infty) \mapsto(-\infty,+\infty)$ is strictly increasing, strictly concave, continuously differentiable, and satisfies the Inada conditions $U^{\prime}\left(0^{+}\right)=+\infty$ and $U^{\prime}(+\infty)=0$.

ii) $\mathcal{C}(x)=\left\{X \in L_{+}^{0} \mid E_{Q}[X] \leq x\right\}$ for $x>0$, where $Q$ is equivalent to $P$.

iii) $\sup _{X \in \mathcal{C}(x)} E_{P}[U(X)]<U(\infty)$

iv) $P$ (and hence $Q$ ) has full support, i.e. $P(G)>0$ for any open set $G$.

i) means that marginal utility spans the whole range $(0, \infty)$. Appetites change smoothly. $i i)$ defines the set of available payoffs in terms on the pricing measure $Q$, and entails that the market is complete. $i i i$ ) is a well-posedness condition. Bliss utility cannot be reached. $i v$ ) means that $\Omega$ includes only relevant events. It does not restrict generality, in that $i v$ ) always holds after replacing $\Omega$ with the support of $P$.

The pricing probability $Q$ identifies each classical payoff $X$ with the finite Borel measure $\mu_{X}=$ $X d Q$, defined by $\mu_{X}(A)=E_{Q}\left[X 1_{A}\right]$. With this identification, the expected utility map $X \mapsto I_{U}(X)$ has the expression:

$$
I_{U}(X):=E_{P}[U(X)]=\int_{\Omega} U\left(\frac{d \mu_{X}}{d Q}(\omega)\right) d P(\omega)=\int_{\Omega} U\left(\frac{d \mu_{X}}{d Q}(\omega)\right) \frac{d P}{d Q}(\omega) d Q(\omega)
$$

Kramkov and Schachermayer (1999) show with counterexamples that the original problem (UM) may not have a solution if $A E(U)=1$. In a complete market, they show that maximizing sequences may entail concentration of capital on "cheap" Arrow-Debreu securities, on which $\frac{d Q}{d P}(\omega) \approx 0$. Such securities, which yield a large payoff $X$ on an event of tiny probability, seem superficially irrelevant for utility maximization, as the marginal utility $U^{\prime}(X)$ decreases to zero for large payoffs. However, as the odds become more attractive, the quantity $U^{\prime}(X) d P / d Q$ may remain bounded. For example, imagine a sequence $\left(\omega_{n}\right)_{n \geq 1}$ converging to some $\omega_{\infty}$, and such that $\left.\lim _{n \uparrow \infty}(d P / d Q)\left(\omega_{n}\right) \uparrow \infty\right)$. Thus, an optimal allocation, where $U^{\prime}(X(\omega))$ is proportional to $(d P / d Q)(\omega)$, may well entail a "payoff" such that $Q\left(\omega_{\infty}\right)>0$, even if $P\left(\omega_{\infty}\right)=0$. See Schachermayer (2002) for a further discussion of this phenomenon. 
This scenario baffles the existing mathematical theory in two ways. First, the utility map loses its upper semicontinuity with respect to maximizing sequences, as the utilities of maximizing payoffs are no longer uniformly integrable. Second, the purely measure theoretic setting $(\Omega, \mathcal{F}, P)$ becomes inadequate to represent singular capital allocations. If a maximizing sequence $\left(X_{n} d Q\right)_{n \geq 0}$ converges to a Dirac delta on some $\omega$, this delta is a natural candidate for a maximizer. On the other hand, if $P(\omega)=0$, removing $\omega$ from the original $\Omega$ leads to an equivalent model where no such candidate exists. Thus, a solution may or may not exist, depending on the initial choice of the sample space $\Omega$.

This paper starts from the observation that in most models the sample space $\Omega$ is already equipped with a topological structure. For example, in diffusion models $\Omega$ is the Wiener space endowed with the uniform topology, while discontinuous models lead to the Skorokhod space. Furthermore, these topologies are compatible with a complete separable metric - they are Polish spaces.

The Polish space structure allows to identify payoffs as measures. This perspective is economically straightforward, thinking of $\Omega$ as a roulette table, and of a payoff as a distribution of chips on the various numbers. The payoffs of the form $\mu_{X}=X d Q$ are a subclass of the norm dual space $\left(C_{b}(\Omega)\right)^{*}$, which is isometric to $r b a(\Omega)$, the space of Borel regular, finitely additive signed measures on $\Omega$ (Dunford and Schwartz, 1988, IV.6). Each element $\mu \in \operatorname{rba}(\Omega)$ admits the unique three-way decomposition:

$$
\mu=\mu_{a}+\mu_{s}+\mu_{p}
$$

where $\mu_{a}$ and $\mu_{s}$ are countably additive measures, respectively absolutely continuous and singular with respect to $P$, and $\mu_{p}$ is a purely finitely additive measure. All three components are Borel regular.

Because $r b a(\Omega)$ is the dual of a Banach space, its bounded sets - including sequences of available payoffs in $\mathcal{C}(x)$ - are relatively weak star compact. This property is crucial, as it yields limits to maximizing sequences.

Definition 1.2. A relaxed payoff is an element of $\mathcal{D}(x)$, the weak star $\sigma\left(r b a(\Omega), C_{b}(\Omega)\right)$ closed set $\left\{\mu \in \operatorname{rba}(\Omega)_{+} \mid \mu(\Omega) \leq x\right\}$.

The disadvantage of $\mathcal{D}(x)$ is to include purely finitely additive measures, which have a dubious interpretation as payoffs. By contrast, countably additive measures - including those singular with respect to $Q$ - allow the usual Arrow-Debreu interpretation of bets paying off in certain states of nature. This paper resolves this issue by allowing a priori all relaxed payoffs, including finitely additive ones. Then, an additional coercivity assumption implies a posteriori that the optimal payoff is countably additive. This assumption ensures that exceptionally favorable states (i.e., where $\varphi$ is high) do not disperse outside the compact sets of $\Omega$.

Defining $\mathcal{D}(x)$ as the set of relaxed payoffs, the relaxed utility map $\overline{I_{U}}: r b a(\Omega) \rightarrow[-\infty,+\infty)$, defined on $\operatorname{rba}(\Omega)$, is the upper semicontinuous envelope of the original $I_{U}$ :

$$
\overline{I_{U}}(\mu)=\inf \left\{G(\mu) \mid G: \operatorname{rba}(\Omega) \rightarrow[-\infty,+\infty), G \text { weak }{ }^{*} \text { u.s.c., } G \geq I_{U} \text { on } L^{1}(Q)\right\}
$$

Since the relaxed utility map $\overline{I_{U}}$ is weak star upper semicontinuous by definition, and the space of relaxed payoffs $\mathcal{D}(x)$ is weak star compact, the relaxed utility maximization problem:

$$
\max _{\mu \in \mathcal{D}(x)} \overline{I_{U}}(\mu)
$$

admits a solution by construction, and the problems (UM) and (RUM) have the same value,

$$
\sup _{X \in \mathcal{C}(x)} I_{U}(X)=\max _{\mu \in \mathcal{D}(x)} \overline{I_{U}}(\mu)
$$


The challenge is to find a "concrete" representation for $\overline{I_{U}}$, i.e. an explicit formula for the relaxed utility map. This task, which is accomplished in Section 2, involves two additional concepts: the singular utility $\varphi$, and the sup-convolution $W$. These concepts in turn rely on the convex conjugates of the utility function $U$ and of the expected utility functional $I_{U}$, which are discussed in the next Section.

The convex conjugate of $U$ is the function $V: \mathbb{R} \rightarrow(-\infty,+\infty]$ defined as $V(y)=\sup _{x>0}(U(x)-$ $x y$ ), so that $V(y)=+\infty$ for $y<0$. The singular utility is defined as the nonnegative function:

$$
\varphi(\omega)=\inf \left\{g(\omega) \mid g \in \mathcal{C}_{b}(\Omega), E_{P}\left[V\left(g \frac{d Q}{d P}\right)<\infty\right]\right\}
$$

which is upper semi-continuous, because it is the infimum of a family of continuous functions. Assumption 1.1 iii) implies that $\varphi$ is finite valued. Indeed, Kramkov and Schachermayer (1999, Theorem $2.0 \mathrm{i})$ ) show that this assumption is equivalent to the existence of some $\widetilde{y}>0$ such that $E_{P}\left[V\left(\widetilde{y} \frac{d Q}{d P}\right)\right]<+\infty$. Thus, $\varphi \leq \widetilde{y} . W: \Omega \times \mathbb{R}_{+} \rightarrow \mathbb{R}$ is defined as the pointwise sup-convolution of the utility function $U$ and of the random function $x \mapsto x \varphi(\omega) \frac{d Q}{d P}(\omega)$ :

$$
W(\omega, x):=\sup _{z \leq x}\left(U(z)+(x-z) \varphi(\omega) \frac{d Q}{d P}(\omega)\right)
$$

The main result on integral representation is then:

Theorem 1.3. Let $\mu \in \operatorname{rba}(\Omega)_{+}$, and let $Q$ be a probability on $\Omega$ with full support.

i) In general:

$$
\overline{I_{U}}(\mu)=E_{P}\left[W\left(\cdot, \frac{d \mu_{a}}{d Q}\right)\right]+\int \varphi d \mu_{s}+\inf _{f \in \operatorname{Dom}\left(J_{V}\right)} \mu_{p}(f)
$$

ii) If $\varphi=0$ P-a.s., then

$$
\overline{I_{U}}(\mu)=E_{P}\left[U\left(\frac{d \mu_{a}}{d Q}\right)\right]+\int \varphi d \mu_{s}+\inf _{f \in \operatorname{Dom}\left(J_{V}\right)} \mu_{p}(f)
$$

iii) If $\lim \sup _{x \uparrow \infty} \frac{x U^{\prime}(x)}{U(x)}<1$, then $\{\varphi=0\}=\Omega$ and

$$
\overline{I_{U}}(\mu)=E_{P}\left[U\left(\frac{d \mu_{a}}{d Q}\right)\right]
$$

This result is understood as follows. The general formula $i$ ) holds for any $\mu \in \operatorname{rba}(\Omega)_{+}$, but does not have a sound economic interpretation, since it involves the finitely additive part $\mu_{p}$ and the sup-convolution $W$, which differs from the original utility function $U$. Formula $i i$ ) resolves the second issue, showing that $W$ boils down to $U$ if $\varphi$ is almost surely null. Example 4.3 in Section 4 shows with a counterexample that $U$ and $W$ may differ without this additional assumption.

Then the relaxed utility is understood as the sum of three parts: the classical expected utility $E[U(X)]$, where $X=\frac{d \mu_{a}}{d Q}$, the singular utility $\int \varphi d \mu_{s}$, and the purely finitely additive term $\mu_{p}$. Intuitively, the singular term accounts for the utility from the concentration of wealth on $P$-null events, in that $\varphi(\omega)$ represents the maximal expected utility from a Dirac delta concentrated at $\omega$. Indeed, $\varphi$ vanishes at each $\omega$ where $d P / d Q$ is locally bounded (i.e. bounded in a neighborhood of $\omega$ ), because concentrating wealth is suboptimal if the odds are finite. On the other hand, concentration of wealth may yield a positive utility $\varphi(\omega)$ at those $\omega$ where $d P / d Q$ is unbounded, that is, when 
the odds are arbitrarily good. The value of $\varphi(\omega)$ depends on the speed at which $d P / d Q$ explodes near $\omega$.

Finally, formula iii) reconciles the theorem with the result of Kramkov and Schachermayer (1999), who show that the utility maximization problem admits a classical solution under the

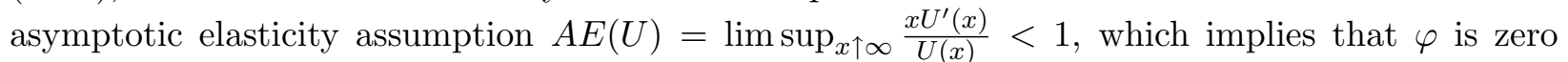
everywhere (and not merely almost). Thus, the additional terms vanish, and the expected utility function depends only on $\mu_{a}=X d Q$.

When $A E(U)=1$, Assumption 1.1 and $\varphi=0$ do not guarantee that any optimizer $\mu^{*}$ of (RUM) is a measure, i.e. $\mu_{p}^{*}=0$. Example 4.4 makes this point with a counterexample. This problem is resolved by the next Assumption, which rules out the purely finitely additive part.

Assumption 1.4. There exist $g \in \operatorname{Dom}\left(J_{V}\right)$ such that the closed set $K=\left\{g \geq y_{0}-\varepsilon\right\}$ is compact for some $\varepsilon>0$, where $y_{0}=\sup _{\omega \in \Omega} \varphi(\omega)$.

To state the main result on utility maximization, define $u$ as the value function of the utility maximization problem (UM)

$$
u(x)=\sup \left\{E_{P}[U(X)] \mid E_{Q}[X] \leq x\right\}
$$

and let $v$ be its conjugate: $v(y)=\sup _{x>0}\{u(x)-x y\}$. Finally, set $x_{0}=\lim _{y \downarrow} y_{0}-v^{\prime}(y)=-v_{+}^{\prime}\left(y_{0}\right)$. Then, $x_{0} \in(0,+\infty]$ is the capital threshold above which the optimal payoff includes a singular component.

Theorem 1.5. If Assumptions 1.1 and 1.4 hold, and $\varphi=0$ a.s., it follows that:

i) $u(x)=\max _{\mu \in \mathcal{D}(x)} \overline{I_{U}}(\mu)$

ii) $\mu^{*}=X^{*} d Q+\mu_{s}^{*}$, and:

$$
u(x)=E\left[U\left(X^{*}\right)\right]+\int \varphi d \mu_{s}^{*}
$$

$X^{*}$ is unique, and the budget constraint is binding: $\mu^{*}(\Omega)=E_{Q}\left[X^{*}\right]+\mu_{s}^{*}(\Omega)=x$. The support of any $\mu_{s}^{*}$ satisfies:

$$
\operatorname{supp}\left(\mu_{s}^{*}\right) \subseteq \operatorname{argmax}(\varphi)
$$

iii) Optimizers depend on the initial capital $x$ as follows:

a) $x \leq x_{0}$ (Kramkov and Schachermayer (1999), Theorem 2.0)

The unique solution is $\mu^{*}=X^{*}(x) d Q$, where $X^{*}(x)=\left(U^{\prime}\right)^{-1}\left(y(x) \frac{d Q}{d P}\right)$ and $y(x)=$ $\left(v^{\prime}\right)^{-1}(-x)$.

b) $x>x_{0}$

Any solution has the form $\mu^{*}=X^{*}(x) d Q+\mu_{s}^{*}$, where $X^{*}(x)=X^{*}\left(x_{0}\right)=\left(U^{\prime}\right)^{-1}\left(y_{0} \frac{d Q}{d P}\right)$ and $\mu_{s}^{*}(\Omega)=x-x_{0}$. Therefore $u(x)=u\left(x_{0}\right)+\left(x-x_{0}\right) \max _{\omega} \varphi(\omega)=u\left(x_{0}\right)+\left(x-x_{0}\right) y_{0}$.

The novelty of this Theorem is the existence of optimal solutions, and their description in the singular case: when $x_{0}$ is finite and $x>x_{0}$, it is optimal to invest the residual capital $x-x_{0}$ in a very unlikely - but also very favorable - bet $\mu_{s}^{*}$. Such bet is not unique in general, because its contribution to expected utility is linear and therefore multiple solutions arise as soon $\operatorname{as} \operatorname{argmax}(\varphi)$ has more than one element. 


\section{Representation of the relaxed functional $\overline{I_{U}}$}

This section proves Theorem 1.3, the representation formula for the relaxed utility map $\overline{I_{U}}$. The argument proceeds in three steps:

i) separate in $\overline{I_{U}}$ the countably additive part from the purely finitely additive part (Lemma 2.2 );

ii) find an integral representation for the countably additive part, separating the absolutely continuous and the singular components with respect to $Q$ (Proposition 2.6);

iii) identify the absolutely continuous part as the original expected utility map, and the singular part as an "asymptotic utility" (Lemma 2.4 and 2.9).

The convex conjugate $J_{V}: \mathcal{C}_{b}(\Omega) \rightarrow(-\infty,+\infty]$ of the expected utility map $I_{U}$ is:

$$
J_{V}(g):=\sup _{X \in L^{1}(Q)}\left(I_{U}(X)-E_{Q}[g X]\right)=E_{P}\left[V\left(g \frac{d Q}{d P}\right)\right]
$$

The domain of $J_{V}$ is defined as Dom $J_{V}=\left\{g \in \mathcal{C}_{b}(\Omega): E[V(g d Q / d P)]<\infty\right\}$ and is a subset of $\mathcal{C}_{b}(\Omega)_{+}$. The next Lemma collects some properties of the conjugate functional $J_{V}$.

\section{Lemma 2.1.}

i) $J_{V}(g)=E_{P}\left[V\left(g \frac{d Q}{d P}\right)\right]$;

ii) $\operatorname{Dom}\left(J_{V}\right)=\left\{g \in \mathcal{C}_{b}(\Omega) \mid E_{P}\left[V\left(g \frac{d Q}{d P}\right)\right]<+\infty\right\}$, the proper domain of $J_{V}$, is contained in $\mathcal{C}_{b}(\Omega)_{+}$and is directed downward;

iii) $\varphi(\omega)=\inf _{g \in \operatorname{Dom}\left(J_{V}\right)} g(\omega)$ defines a random variable, which is positive, bounded, and upper semicontinuous. In addition, there exists a decreasing sequence $\left(g_{k}\right)_{k \geq 1} \subset \operatorname{Dom}\left(J_{V}\right)$ such that $g_{k}(\omega) \downarrow \varphi(\omega)$ for all $\omega$.

Proof. $L^{1}(Q)$ is decomposable (i.e. $f 1_{A}+g 1_{\Omega \backslash A} \in L^{1}(Q)$ for any $f, g \in L^{1}(Q)$ and $A \in \mathcal{F}$ ), therefore i) follows from (Rockafellar, 1974, Theorem 21, part a)). Since $\operatorname{Dom}(V) \subseteq \mathbb{R}_{+}$, then $\operatorname{Dom}\left(J_{V}\right) \subset$ $\mathcal{C}_{b}(\Omega)_{+}$. Thus, the pointwise infimum $\varphi$ of the family of continuous, bounded, nonnegative functions $\operatorname{Dom}\left(J_{V}\right)$ is well-defined, nonnegative, bounded and upper semicontinuous. Also, $\operatorname{Dom}\left(J_{V}\right)$ is directed downward, because $g \wedge f \in \operatorname{Dom}\left(J_{V}\right)$ if $g, f \in \operatorname{Dom}\left(J_{V}\right)$ :

$$
E_{P}\left[V\left(g \wedge f \frac{d Q}{d P}\right)\right]=E_{P}\left[V\left(g \frac{d Q}{d P}\right) 1_{\{g \leq f\}}\right]+E_{P}\left[V\left(f \frac{d Q}{d P}\right) 1_{\{f<g\}}\right]<+\infty
$$

Moreover, the space $\mathcal{C}_{b}(\Omega)$ has the countable supremum property (Aliprantis and Border, 2006, Theorem 8.22). This combined with the directed-downward property implies the existence of a monotone sequence $\left(g_{k}\right)_{k \geq 1}$ in $\operatorname{Dom}\left(J_{V}\right)$ such that $g_{k} \geq \varphi$ and $g_{k} \downarrow \varphi$ pointwise.

An application of Hahn-Banach separation theorem (see e.g. Borwein and Lewis (2006, Theorem 4.2.8) ensures that the relaxation $\overline{I_{U}}$ coincides with the biconjugate functional $\left(I_{U}\right)^{* *}: r b a(\Omega) \rightarrow$ $[-\infty,+\infty)$, which is defined as:

$$
\left(I_{U}\right)^{* *}(\mu)=\inf _{g \in \mathcal{C}_{b}(\Omega)}\left(\mu(g)+E_{P}\left[V\left(g \frac{d Q}{d P}\right)\right]\right)
$$


The infimum over $\mathcal{C}_{b}(\Omega)$ in the above formula can be replaced by the infimum over $\operatorname{Dom}\left(J_{V}\right)$, since by $(11),\left(I_{U}\right)^{* *}=-\infty$ whenever $\mu$ is not positive. The results in the rest of the Section are stated only for $\mu \in r b a_{+}$.

The following Lemma proves the first part of Theorem 1.3, which states that the relaxation is additive across the Yosida and Hewitt (1952) decomposition of $\mu=\mu_{c}+\mu_{p}$ in terms of the countably additive part $\mu_{c}=\mu_{a}+\mu_{s}$, and the purely finitely additive part $\mu_{p}$. Since $\Omega$ is a Polish space, any measure $\mu=\mu_{c} \in \operatorname{rba}(\Omega)_{+}$is a Radon measure, that is compact-inner regular (Aliprantis and Border, 2006, Theorem 12.7). By contrast, any purely finitely additive $\mu=\mu_{p}$ vanishes on compact sets (Aliprantis and Border, 2006, Theorem 12.4). This contrasting behavior allows the separation of the contributes of $\mu_{c}$ and $\mu_{p}$ in the relaxation (12).

Lemma 2.2. Let $\mu \in \operatorname{rba}(\Omega)_{+}$. Then

$$
\overline{I_{U}}(\mu)=\overline{I_{U}}\left(\mu_{c}\right)+\inf _{f \in \operatorname{Dom}\left(J_{V}\right)} \mu_{p}(f)
$$

Proof. The inequality $\geq$ follows from $\overline{I_{U}}=\left(I_{U}\right)^{* *}$ and from the inequality

$E_{P}\left[V\left(g \frac{d Q}{d P}\right)\right]+\mu(g) \geq E_{P}\left[V\left(g \frac{d Q}{d P}\right)\right]+\mu_{c}(g)+\inf _{f \in \operatorname{Dom}\left(J_{V}\right)} \mu_{p}(f) \geq\left(I_{U}\right)^{* *}\left(\mu_{c}\right)+\inf _{f \in \operatorname{Dom}\left(J_{V}\right)} \mu_{p}(f)$

For the opposite inequality, note that $\left(P+\mu_{c}\right)$ is a Radon measure. Hence there exists an increasing sequence of compact sets $K^{n}$ such that $\left(P+\mu_{c}\right)\left(\Omega \backslash K^{n}\right)<\frac{1}{n}$. By contrast, $\mu_{p}\left(K^{n}\right)=0$ for all $n$ because $\mu_{p}$ is purely finitely additive. Thus $\mu_{p}$ is concentrated on $\Omega \backslash K^{n}$. Borel-regularity of $\mu_{p}$ implies the existence of closed sets $C^{n} \subseteq \Omega \backslash K^{n}$ such that

$$
\mu_{p}\left(\Omega \backslash C^{n}\right)<\frac{1}{n}
$$

In the Polish space $\Omega$, closed sets are separated by continuous functions. That is, there exists a continuous function $\alpha^{n}: \Omega \rightarrow[0,1]$ which is equal to 1 on $K^{n}$ and 0 on $C^{n}$. In fact, if $d$ is a distance that induces the topology $\mathcal{T}$ on $\Omega$, one such function is:

$$
\alpha^{n}(\omega)=\frac{d\left(\omega, C^{n}\right)}{d\left(\omega, C^{n}\right)+d\left(\omega, K^{n}\right)}
$$

Up to a subsequence, $\alpha^{n}$ converges to $1\left(P+\mu_{c}\right)$-a.s. Fix an some $f, g \in \operatorname{Dom}\left(J_{V}\right)$, and set

$$
h^{n}=\alpha^{n} g+\left(1-\alpha^{n}\right) f
$$

Convexity of $V$ and boundedness of $\alpha^{n}$ imply $h^{n} \in \operatorname{Dom}\left(J_{V}\right)$, because

$$
E_{P}\left[V\left(h^{n} \frac{d Q}{d P}\right)\right]+\mu\left(h^{n}\right) \leq E_{P}\left[\alpha^{n} V\left(g \frac{d Q}{d P}\right)\right]+E_{P}\left[\left(1-\alpha^{n}\right) V\left(f \frac{d Q}{d P}\right)\right]+\mu\left(h^{n}\right)<+\infty
$$

Also, since $h^{n}-f=\alpha^{n}(g-f)$, and $0 \leq \alpha^{n} \leq 1$

$$
\mu_{p}\left(h^{n}\right) \leq \frac{1}{n}\|g-f\|_{\infty}+\mu_{p}(f)
$$

It follows that

$$
\begin{aligned}
\left(I_{U}\right)^{* *}(\mu) & \leq E_{P}\left[V\left(h^{n} \frac{d Q}{d P}\right)\right]+\mu\left(h^{n}\right) \\
& \leq E_{P}\left[\alpha^{n} V\left(g \frac{d Q}{d P}\right)\right]+E_{P}\left[\left(1-\alpha^{n}\right) V\left(f \frac{d Q}{d P}\right)\right]+\mu_{c}\left(h^{n}\right)+\frac{1}{n}\|g-f\|_{\infty}+\mu_{p}(f)
\end{aligned}
$$


and passing to the liminf,

$$
\begin{aligned}
\left(I_{U}\right)^{* *}( & \mu \\
& \leq \liminf _{n \uparrow \infty} E_{P}\left[V\left(h^{n} \frac{d Q}{d P}\right)\right]+\mu\left(h^{n}\right) \\
\leq & \lim _{n \uparrow \infty}\left\{E_{P}\left[\alpha^{n} V\left(g \frac{d Q}{d P}\right)\right]+E_{P}\left[\left(1-\alpha^{n}\right) V\left(f \frac{d Q}{d P}\right)\right]+\mu_{c}\left(h^{n}\right)+\frac{1}{n}\|g-f\|_{\infty}+\mu_{p}(f)\right\} \\
= & E_{P}\left[V\left(g \frac{d Q}{d P}\right)\right]+\mu_{c}(g)+\mu_{p}(f)
\end{aligned}
$$

where the liminf in the second line becomes a limit, because $\alpha^{n}$ is bounded and converges to 1 $\left(P+\mu_{c}\right)$-a.s., hence the dominated convergence theorem applies. Thus:

$$
\left(I_{U}\right)^{* *}(\mu) \leq \inf _{f, g \in \operatorname{Dom}\left(J_{V}\right)}\left(E_{P}\left[V\left(g \frac{d Q}{d P}\right)\right]+\mu_{c}(g)+\mu_{p}(f)\right)=\left(I_{U}\right)^{* *}\left(\mu_{c}\right)+\inf _{f \in \operatorname{Dom}\left(J_{V}\right)} \mu_{p}(f)
$$

which completes the proof.

Remark 2.3. It is tempting to replace the expression $\inf _{f \in \operatorname{Dom}\left(J_{V}\right)} \mu_{p}(f)$ with the simpler $\mu_{p}(\varphi)$, i.e exchange the infimum and the expectation. However, since $\mu_{p}$ is not countably additive, only the inequality $\mu_{p}(\varphi) \leq \inf _{f \in \operatorname{Dom}\left(J_{V}\right)} \mu_{p}(f)$ holds in general. Example 4.4 shows a situation where $\varphi=0$ but inf $_{f \in \operatorname{Dom}\left(J_{V}\right)} \mu_{p}(f)>0$.

Denote the countably additive elements of $r b a(\Omega)_{+}$simply by $\mathcal{M}_{+}$, the subset of positive Radon measures. The next step is to prove an integral representation for $\overline{I_{U}}(\mu)$ when $\mu \in \mathcal{M}_{+}$. This result extends in part the work of Bouchitté and Valadier (1988), who consider a locally compact space $\Omega$. Relaxing this assumption is central in Mathematical Finance where sample spaces are typically infinite-dimensional.

Recall the definition of $W: \Omega \times \mathbb{R}_{+} \rightarrow \mathbb{R}$, the $\omega$-wise sup-convolution of the utility function $U$ and of the random function $x \mapsto x \varphi(\omega) \frac{d Q}{d P}(\omega)$ :

$$
W(\omega, x):=\sup _{z \leq x}\left(U(z)+(x-z) \varphi(\omega) \frac{d Q}{d P}(\omega)\right)
$$

The sup-convolution $W$ may differ from $U$ only on the event $\left\{\varphi \frac{d Q}{d P}>0\right\}$ :

Lemma 2.4. $\{\omega \mid W(\omega, x)=U(x)$ for all $x>0\}=\left\{\omega \mid \varphi(\omega) \frac{d Q}{d P}(\omega)=0\right\}$.

Proof. If $\varphi(\omega) \frac{d Q}{d P}(\omega)=0$, then $W(\omega, x)=U(x)$ from the definition of $W$. Viceversa, observe that if $W(\omega, x)=U(x)$ for all $x>0$, then:

$$
U^{\prime}(z)-\varphi(\omega) \frac{d Q}{d P}(\omega) \geq 0 \quad \text { for all } z>0
$$

and the claim follows from the Inada condition $U^{\prime}(\infty)=0$ :

$$
0=\lim _{z \rightarrow+\infty} U^{\prime}(z) \geq \varphi(\omega) \frac{d Q}{d P}(\omega)
$$


Lemma 2.5. If $\mu \in \mathcal{M}_{+}$, then

$$
\overline{I_{U}}(\mu)=\sup _{X_{n} \stackrel{*}{\rightarrow} \mu} \limsup _{n \uparrow \infty} I_{U}\left(X_{n}\right)
$$

where the maximum is taken over all sequences $\left(X_{n}\right)_{n}$ that weak star converge to $\mu$.

Proof. The relaxation $\overline{I_{U}}$ is defined as the upper semicontinuous envelope of $I_{U}$, hence (cf. Buttazzo (1989, Proposition 1.3.1)):

$$
\overline{I_{U}}(\mu)=\sup _{X_{\alpha} \stackrel{*}{\rightarrow} \mu} \lim \sup I_{U}\left(X_{\alpha}\right)
$$

where the supremum is taken over all nets $\left(X_{\alpha}\right)_{\alpha \in I}$ converging weak star to $\mu$. Since the trace of the weak star topology on norm bounded subset of $\mathcal{M}_{+}$is metrizable (e.g. by the Dudley distance, cf. Ambrosio, Gigli and Savaré (2008, Section 5.1)), nets can be replaced by sequences for $\mu \in \mathcal{M}_{+}$.

Proposition 2.6. Let $\mu \in \mathcal{M}_{+}$, so that $\mu=\mu_{c}=\mu_{a}+\mu_{s}$. Then

$$
\overline{I_{U}}(\mu)=E_{P}\left[W\left(\cdot, \frac{d \mu_{a}}{d Q}\right)\right]+\int \varphi d \mu_{s}
$$

Proof. By (11) and Lemma 2.5, the relaxed functional satisfies:

$$
\overline{I_{U}}(\mu)=\sup _{X_{n} * \mu} \limsup _{n \uparrow \infty} I_{U}\left(X_{n}\right)=\left(I_{U}\right)^{* *}(\mu)=\inf _{g \in \mathcal{C}_{b}(\Omega)}\left(\mu(g)+E_{P}\left[V\left(g \frac{d Q}{d P}\right)\right]\right)
$$

Consider a maximizing sequence $\left(X_{n}\right)_{n \geq 1}$ for $\overline{I_{U}}(\mu)$. As $\mu^{n}:=X_{n} d Q$ converges to $\mu$ in the weak star topology, $\left(X_{n}\right)_{n}$ is bounded in $L^{1}(Q)$. Up to a sequence of convex combinations, which preserves the maximizing property by concavity of $I_{U}$, Komlos Theorem implies that $\left(X_{n}\right)_{n>1}$ converges $Q$-a.s. to some positive random variable $Z$. Lemma 2.7 below implies that $Z \leq \frac{d \mu_{a}}{d Q}$. For any $g \in \operatorname{Dom}\left(J_{V}\right)$, the pointwise Fenchel inequality $U(x) \leq x y+V(y)$ yields:

$$
U\left(X_{n}\right)-X_{n} g \frac{d Q}{d P} \leq V\left(g \frac{d Q}{d P}\right)
$$

Passing to the limsup of the expectations, Fatou's Lemma implies that:

$$
\underset{n \uparrow \infty}{\limsup } E_{P}\left[U\left(X_{n}\right)-X_{n} g \frac{d Q}{d P}\right] \leq E_{P}\left[U(Z)-Z g \frac{d Q}{d P}\right] \leq E_{P}\left[V\left(g \frac{d Q}{d P}\right)\right]
$$

Since $\left(X_{n}\right)_{n \geq 1}$ is maximizing, and $E\left[X_{n} g \frac{d Q}{d P}\right]=E_{Q}\left[X_{n} g\right]$ converges to $\mu(g)$,

$$
\overline{I_{U}}(\mu)-\mu(g) \leq E_{P}\left[U(Z)-Z g \frac{d Q}{d P}\right] \leq E_{P}\left[V\left(g \frac{d Q}{d P}\right)\right]
$$

or equivalently, adding $\mu(g)$ to all members above and decomposing $\mu=\mu_{a}+\mu_{s}$

$$
\overline{I_{U}}(\mu) \leq E_{P}\left[U(Z)+\left(\frac{d \mu_{a}}{d Q}-Z\right) g \frac{d Q}{d P}\right]+\mu_{s}(g) \leq E_{P}\left[V\left(g \frac{d Q}{d P}\right)\right]+\mu(g)
$$

which holds for any $g \in \operatorname{Dom}\left(J_{V}\right)$. Take now the infimum on $g$ in the above chain. Lemma 2.1 iii) and Monotone Convergence Theorem ensure that the infimum can be taken within the expectation signs in the middle term. Then

$$
\overline{I_{U}}(\mu) \leq E_{P}\left[U(Z)+\left(\frac{d \mu_{a}}{d Q}-Z\right) \varphi \frac{d Q}{d P}\right]+\mu_{s}(\varphi) \leq\left(I_{U}\right)^{* *}(\mu)
$$


which implies that both inequalities are in fact equalities. Thus, it remains to prove that:

$$
E_{P}\left[U(Z)+\left(\frac{d \mu_{a}}{d Q}-Z\right) \varphi \frac{d Q}{d P}\right]=E_{P}\left[W\left(\cdot, \frac{d \mu_{a}}{d Q}\right)\right]
$$

For any $g \in \operatorname{Dom}\left(J_{V}\right)$ and $0 \leq z \leq x$ :

$$
U(z)+(x-z) \varphi \frac{d Q}{d P} \leq U(z)+(x-z) g \frac{d Q}{d P} \leq V\left(g \frac{d Q}{d P}\right)+x g \frac{d Q}{d P}
$$

where the first inequality is due to $\varphi \leq g$ and the second is an application of Fenchel inequality, $U(z)-z y \leq V(y)$. Therefore:

$$
U(z)+(x-z) \varphi \frac{d Q}{d P} \leq W(\omega, x)=\sup _{z \leq x}\left(U(z)+(x-z) \varphi \frac{d Q}{d P}\right) \leq V\left(g \frac{d Q}{d P}\right)+x g \frac{d Q}{d P}
$$

Substituting $x$ with $\frac{d \mu_{a}}{d Q}, z$ with $Z$ in the first term on the left, and taking expectations:

$$
E_{P}\left[U(Z)+\left(\frac{d \mu_{a}}{d Q}-Z\right) \varphi \frac{d Q}{d P}\right] \leq E_{P}\left[W\left(\cdot, \frac{d \mu_{a}}{d Q}\right)\right] \leq E_{P}\left[V\left(g \frac{d Q}{d P}\right)\right]+\mu_{a}(g)
$$

Thus, combining (16) with (15), the following holds for any $g \in \operatorname{Dom}\left(J_{V}\right)$ :

$$
\begin{aligned}
\overline{I_{U}}(\mu) & =E_{P}\left[U(Z)+\left(\frac{d \mu_{a}}{d Q}-Z\right) \varphi \frac{d Q}{d P}\right]+\mu_{s}(\varphi) \\
& \leq E_{P}\left[W\left(\cdot, \frac{d \mu_{a}}{d Q}\right)\right]+\mu_{s}(\varphi) \leq E_{P}\left[V\left(g \frac{d Q}{d P}\right)\right]+\mu_{a}(g)+\mu_{s}(g)
\end{aligned}
$$

whence the conclusion (14). Moreover $U(Z)+\left(\frac{d \mu_{a}}{d Q}-Z\right) \varphi \frac{d Q}{d P}=W\left(\cdot, \frac{d \mu_{a}}{d Q}\right)$ almost surely, whence the pointwise limit $Z$ of the maximizing $\left(X_{n}\right)_{n}$ verifies

$$
Z=\frac{d \mu_{a}}{d Q} \wedge\left(U^{\prime}\right)^{-1}\left(\varphi \frac{d Q}{d P}\right) \text { a.s. }
$$

Lemma 2.7. Let $\left(X_{n}\right)_{n \geq 1}$ be a bounded sequence in $L_{+}^{1}(Q)$, such that $X_{n}$ converges to $X$ almost surely, and weak star to $\mu \in \operatorname{rba}(\Omega)$. Then $X \leq \frac{d \mu_{a}}{d Q}$ almost surely.

Proof. Note first that $\mu \geq 0, X \geq 0$ and $X \in L^{1}(Q)$ by Fatou's Lemma. By the compact-inner regularity of the measure $\mu_{a}+\mu_{s}$, it suffices to show that:

$$
E_{Q}\left[I_{K} X\right] \leq\left(\mu_{a}+\mu_{s}\right)(K) \text { for all compact sets } K
$$

Indeed, since the inequality for all compact sets $K$, it also holds for all Borel sets $B$, whence $E_{Q}\left[I_{B} X\right] \leq\left(\mu_{a}+\mu_{s}\right)(B)$, and in particular $X \leq \frac{d \mu_{a}}{d Q} Q$-a.s.

To this end, proceed similarly to the first part of the proof of Proposition 2.2. Consider a compact $K$. For any $h \geq 1$ there exists a closed set $C_{h} \subseteq K^{c}$ with $\mu_{p}\left(C_{h}\right) \geq \mu_{p}(\Omega)-\frac{1}{h}$. Also, there is a continuous function $g_{h}^{K}$ such that $0 \leq g_{h}^{K} \leq 1, g_{h}^{K}=1$ on $K, g_{h}^{K}=0$ on $C_{h}$ and hence, as $h \uparrow+\infty, g_{h}^{K} \rightarrow 1_{K}$ pointwise. Then, for all $h \geq 1$ :

$$
E_{Q}\left[I_{K} X\right] \leq E_{Q}\left[g_{h}^{K} X\right] \leq \lim _{n \uparrow \infty} E_{Q}\left[g_{h}^{K} X_{n}\right]=\mu\left(g_{h}^{K}\right)
$$


where the second inequality is a consequence of Fatou's Lemma, while the equality follows from weak star convergence of $X_{n} d Q$ to $\mu$. By construction, $\mu_{p}\left(g_{h}^{K}\right) \leq \mu_{p}\left(\Omega \backslash C_{h}\right) \leq \frac{1}{h}$ whence

$$
E_{Q}\left[I_{K} X\right] \leq\left(\mu_{a}+\mu_{s}\right)\left(g_{h}^{K}\right)+\frac{1}{h}
$$

and the conclusion follows passing to the limit as $h \uparrow \infty$.

Remark 2.8. The inequality $X \leq \frac{d \mu_{a}}{d Q}$ can be strict. Ball and Murat (1989, Example 2) give an example in which $X=0$ and $\frac{d \mu_{a}}{d Q}=1$.

It only remains now to put the pieces together.

Proof of Theorem 1.3. $i$ ) follows from Lemma 2.2 and Proposition 2.6. $\varphi=0$ a.s. implies that $W(\omega, x)=U(x)$ almost surely, whence $i i)$ follows from $i$ ) and Lemma 2.4.

To show iii), recall that if $A E(U)$ holds, $E_{P}[V(y d Q / d P)]<+\infty$ for all constants $y>0$ (Kramkov and Schachermayer, 2003, Note 2). Then $\varphi=0$ everywhere on $\Omega$, hence both $\int \varphi d \mu_{s}$ and $\inf _{f \operatorname{Dom}\left(J_{V}\right)} \mu_{p}(f)$ vanish:

$$
\inf _{f \operatorname{Dom}\left(J_{V}\right)} \mu_{p}(f) \leq \inf _{y>0} \mu_{p}(y)=\mu_{p}(\Omega) \inf _{y>0} y=0
$$

Denote by $F \subset \Omega$ the set where $\frac{d P}{d Q}$ is essentially locally bounded (i.e. in a neighborhood):

$$
F:=\left\{\omega \mid \operatorname{ess}_{\sup _{\omega^{\prime}} \in U} \frac{d P}{d Q}\left(\omega^{\prime}\right)<\infty \text { for some open } U \ni \omega\right\}
$$

The complementary set $F^{c}:=\Omega \backslash F$ is the set of the poles of $\frac{d P}{d Q}$, the points at which $\frac{d P}{d Q}$ is unbounded. By definition, $F$ is open, so $F^{c}$ is closed. The following proposition shows that $\varphi$ may be positive only on poles.

Lemma 2.9. $F \subset\{\varphi=0\}$ hence $\{\varphi>0\} \subset F^{c}$.

Proof. If $\omega^{*} \in F$, there exists an open ball $B\left(\omega^{*}, \varepsilon\right) \subset F$ such that $\frac{d P}{d Q} \leq m$ a.s. on $B\left(\omega^{*}, \varepsilon\right)$. Consider $\widetilde{y}>0$ large enough, so that $E_{P}\left[V\left(\widetilde{y} \frac{d Q}{d P}\right)\right]<\infty$, and for any $y \in(0, \widetilde{y})$ consider the continuous bounded function $g_{y}=y \alpha+\widetilde{y}(1-\alpha)$, where

$$
\alpha(\omega)=\frac{d\left(\omega, \Omega \backslash B\left(\omega^{*}, \varepsilon\right)\right)}{d\left(\omega, \bar{B}\left(\omega^{*}, \frac{\varepsilon}{2}\right)\right)+d\left(\omega, \Omega \backslash B\left(\omega^{*}, \varepsilon\right)\right)}
$$

Since $\alpha \in[0,1]$ by construction $y \leq g_{y}<\widetilde{y}$. In addition, $g_{y}(\omega)=y$ for $\omega \in \bar{B}\left(\omega^{*}, \varepsilon / 2\right)$ and $g_{y}(\omega)=\widetilde{y}$ for $\omega \in \Omega \backslash B\left(\omega^{*}, \varepsilon\right)$. To prove that $g_{y} \in \operatorname{Dom}\left(J_{V}\right)$, split the integral $J_{V}(g)=E\left[V\left(g_{y} \frac{d Q}{d P}\right)\right]$ as:

$$
E\left[V\left(g_{y} \frac{d Q}{d P}\right) I_{B\left(\omega^{*}, \varepsilon\right)}\right]+E\left[V\left(g_{y} \frac{d Q}{d P}\right) I_{\Omega \backslash B\left(\omega^{*}, \varepsilon\right)}\right] \leq V\left(\frac{y}{m}\right) P\left(B\left(\omega^{*}, \varepsilon\right)\right)+V(\widetilde{y}) P\left(\Omega \backslash B\left(\omega^{*}, \varepsilon\right)\right)
$$

where the inequality holds since $V$ is decreasing and $g_{y} \frac{d Q}{d P} \geq \frac{y}{m}$ on $B\left(\omega^{*}, \varepsilon\right)$. By definition of $\varphi$ :

$$
\varphi\left(\omega^{*}\right)=\inf _{g \in \operatorname{Dom}\left(J_{V}\right)} g\left(\omega^{*}\right)
$$

and from $g_{y}\left(\omega^{*}\right)=y$, the conclusion $\varphi\left(\omega^{*}\right)=0$ follows.

Corollary 2.10. If $P\left(F^{c}\right)=0$, then $\varphi=0$ a.s. 


\section{Proof of Theorem 1.5}

Recall that the function $\mathbb{R}_{+} \ni y \mapsto v(y)=E\left[V\left(y \frac{d Q}{d P}\right)\right]$ is the restriction of $J_{V}$ to the constant functions and recall that $x_{0}=\lim _{y \downarrow} y_{0}-v^{\prime}(y)=-v_{+}^{\prime}\left(y_{0}\right)$. The next Lemma shows an alternative characterization of $u(x)$ :

Lemma 3.1.

$$
\inf _{g \in \mathcal{C}_{b}(\Omega)}\left(J_{V}(g)+\|g\|_{\infty} x\right)=\inf _{y>0}(x y+v(y))=u(x)
$$

Proof. Only the left equality needs a proof, the other one following from Kramkov and Schachermayer (1999). The inequality $\leq$ is obvious. To see the reverse inequality, observe that $\operatorname{Dom}\left(J_{V}\right) \subseteq$ $\mathcal{C}_{b}(\Omega)_{+}$and $V$ is decreasing. Thus, for all $g \geq 0$

$$
J_{V}(g)+x\|g\|_{\infty} \geq v\left(\|g\|_{\infty}\right)+x\|g\|_{\infty}
$$

which completes the proof.

Lemma 3.2. Let $\mathcal{D}(x)=\left\{\mu \in \operatorname{rba}(\Omega)_{+} \mid \mu(\Omega) \leq x\right\}$, and let $\delta_{\mathcal{D}(x)}$ be the indicator of $\mathcal{D}(x)$. Then its conjugate and biconjugate satisfy:

$$
\begin{aligned}
\left(\delta_{\mathcal{D}(x)}\right)^{*}(g) & =\sup _{\mu \in \operatorname{rba}(\Omega)}\left\{\mu(g)-\delta_{\mathcal{D}(x)}\right\}=\left\|g^{+}\right\|_{\infty} x \\
\left(\delta_{\mathcal{D}(x)}\right)^{* *}(\mu) & =\delta_{\mathcal{D}(x)}(\mu)
\end{aligned}
$$

Proof. As $\mu(g) \leq \mu\left(g^{+}\right)$for any positive $\mu$, the supremum in the formula for the conjugate is reached on the $\mu$ in $\mathcal{D}(x)$ with support contained in $\{g \geq 0\}$. Thus, without loss of generality suppose $g \geq 0$. The inequality $\left(\delta_{\mathcal{D}(x)}\right)^{*}(g) \leq\|g\|_{\infty} x$ follows from the definition of $\mathcal{D}(x)$. To show that equality holds, fix an arbitrary $\epsilon>0$. The upper level set $A=\left\{g>\|g\|_{\infty}-\epsilon\right\}$ is open. Since $Q$ has full support, $Q(A)>0$. Then $\widetilde{\mu}=x \frac{1_{A}}{Q(A)} d Q \in \mathcal{C}(x) \subseteq \mathcal{D}(x)$ and $\widetilde{\mu}(g)>\left(\|g\|_{\infty}-\epsilon\right)$, whence $\left(\delta_{\mathcal{D}(x)}\right)^{*}(g) \geq\left(\|g\|_{\infty}-\epsilon\right)$ for all $\epsilon$.

The original convex functional $\delta_{\mathcal{D}(x)}$ is already weak star lower semicontinuous, because $\mathcal{D}(x)$ is weak star closed. Therefore it coincides with its lower semicontinuous envelope $\left(\delta_{\mathcal{D}(x)}\right)^{* *}$.

Assumption 1.4 is used for the first time in the Lemma below.

Lemma 3.3. If Assumption 1.4 holds, then $\operatorname{argmax} \varphi$ is compact, and:

$$
y_{0}=\max _{\omega \in \Omega} \varphi(\omega)
$$

Proof. Set $c=\inf _{k}\left\|g_{k}\right\|_{\infty}$, where $\left(g_{k}\right)_{k \geq 1}$ decreases to $\varphi$, which exists by Lemma 2.1 iii). As shown in (18), $\left\|g_{k}\right\|_{\infty} \in \operatorname{Dom}\left(J_{V}\right)$ for all $k$, so

$$
c \geq y_{0} \geq \sup _{\omega \in \Omega} \varphi(\omega)
$$

where the last inequality follows from the definitions of $y_{0}$ and $\varphi$. To prove (19), we show that $c=\max \varphi$. Up to replacing $\left(g_{k}\right)_{k \geq 1}$ with $\left(g \wedge g_{k}\right)_{k \geq 1}$, assume that the $g$ in Assumption 1.4 is one of the $g_{k}$, say $g_{k^{*}}$. Then there is a compact upper level of $g_{k^{*}}$ of the form $K=\left\{g_{k^{*}} \geq y_{0}-\varepsilon^{*}\right\}$. As $c \geq y_{0}, K$ contains the closed set $K^{*}=\left\{g_{k^{*}} \geq c-\varepsilon^{*}\right\}$, which is in turn compact. Outside $K^{*}$

$$
\varphi \leq g_{k^{*}}<c-\varepsilon^{*}
$$


As $K^{*}$ is compact and $\varphi$ is u.s.c., it attains its maximum on $K^{*} . K^{*}$ contains all the non empty, closed sets with the finite intersection property: $V_{k, \epsilon}=\left\{g_{k} \geq c-\epsilon\right\}$ for all $k \geq k^{*}, \epsilon<\varepsilon^{*}$. Therefore their intersection $Y:=\bigcap_{k, \epsilon} V_{k, \epsilon}$ is not empty, compact and consists of all the points $\omega^{*}$ where $\lim _{k} g_{k}\left(\omega^{*}\right)=c$. But $\lim _{k} g_{k}\left(\omega^{*}\right)=\varphi\left(\omega^{*}\right)$, so $c=y_{0}=\max _{\omega \in \Omega} \varphi$ and $Y=\operatorname{argmax} \varphi$.

Proof of Theorem 1.5. i): It suffices to show that:

$$
\inf _{g \in \mathcal{C}_{b}(\Omega)}\left(J_{V}(g)+\|g\|_{\infty} x\right)=\max _{\mu \in \mathcal{D}(x)} \overline{I_{U}}(\mu)
$$

Then the claim follows from Lemma 3.1 and the duality formula $u(x)=\inf _{y>0}\{x y+v(y)\}$ (cf. (Kramkov and Schachermayer, 1999, Theorem 2.0)). Since $\operatorname{Dom}\left(J_{V}\right) \subseteq \mathcal{C}_{b}(\Omega)_{+}$,

$$
\inf _{g \in \mathcal{C}_{b}(\Omega)}\left\{J_{V}(g)+\|g\|_{\infty} x\right\}=\inf _{g \in \mathcal{C}_{b}(\Omega)}\left\{J_{V}(g)+\left\|g^{+}\right\|_{\infty} x\right\}=\inf _{g \in \mathcal{C}_{b}(\Omega)}\left\{J_{V}(g)+\left(\delta_{\mathcal{D}(x)}\right)^{*}(g)\right\}
$$

where the last equality follows by Lemma 3.2.

This Lemma and the Fenchel Theorem (Brezis, 1983, Chapter 1) yield the identity:

$$
\inf _{g \in \mathcal{C}_{b}(\Omega)}\left(J_{V}(g)+\left(\delta_{\mathcal{D}(x)}\right)^{*}(g)\right)=\max _{\mu \in \mathcal{D}(x)} \overline{I_{U}}(\mu)
$$

In fact, the Fenchel Theorem implies that

$$
\inf _{g \in \mathcal{C}_{b}(\Omega)}\left(J_{V}(g)+\left(\delta_{\mathcal{D}(x)}\right)^{*}(g)\right)=\max _{\mu \in \operatorname{rba}(\Omega)}\left(-\left(J_{V}\right)^{*}(-\mu)-\left(\delta_{\mathcal{D}(x)}\right)^{* *}(\mu)\right)
$$

Now, by definition $\left(J_{V}\right)^{*}(\mu)=\sup _{g \in \mathcal{C}_{b}(\Omega)}\left\{\mu(g)-J_{V}(g)\right\}$ and thus $\left.-\left(J_{V}\right)^{*}(-\mu)\right)=\left(I_{U}\right)^{* *}(\mu)=$ $\overline{I_{U}}(\mu)$, while $\left(\delta_{\mathcal{D}(x)}\right)^{* *}(\mu)=\delta_{\mathcal{D}(x)}(\mu)$.

ii): The constraint is binding because $\overline{I_{U}}$ is monotone. To prove that any optimal $\mu^{*}$ must be a measure, consider the formula

$$
\overline{I_{U}}\left(\mu^{*}\right)=E_{P}\left[U\left(\frac{d \mu_{a}^{*}}{d Q}\right)\right]+\int \varphi d \mu_{s}^{*}+\inf _{f \in \operatorname{Dom}\left(J_{V}\right)} \mu_{p}^{*}(f)
$$

Suppose that $\mu_{p}^{*} \neq 0$, say $0<\mu_{p}^{*}(\Omega)=x^{\prime} \leq x$. Using (20), the contribution of the purely finitely additive $\mu_{p}^{*}$ to the (optimal) value $\overline{I_{U}}\left(\mu^{*}\right)$ is bounded above by:

$$
\inf _{f \in \operatorname{Dom}\left(J_{V}\right)} \mu_{p}^{*}(f) \leq \mu_{p}^{*}\left(g_{k^{*}}\right)=\mu_{p}^{*}\left(g_{k^{*}} I_{\Omega \backslash K^{*}}\right) \leq\left(y_{0}-\varepsilon^{*}\right) x^{\prime}
$$

Thus a redistribution of capital, e.g. the measure $\widetilde{\mu}=\mu_{a}^{*}+\mu_{s}^{*}+x^{\prime} \nu_{s}$, where $\nu_{s}$ is any probability with support contained in the set $\operatorname{argmax} \varphi$, gives a higher utility:

$$
\begin{aligned}
\overline{I_{U}}(\widetilde{\mu}) & =E_{P}\left[U\left(\frac{d \mu_{a}^{*}}{d Q}\right)\right]+\int \varphi d\left(\mu_{s}^{*}+x^{\prime} \nu_{s}\right) \\
& =E_{P}\left[U\left(\frac{d \mu_{a}^{*}}{d Q}\right)\right]+\int \varphi d \mu_{s}^{*}+y_{0} x^{\prime} \geq \overline{I_{U}}\left(\mu^{*}\right)+\varepsilon^{*} x^{\prime}>\overline{I_{U}}\left(\mu^{*}\right)
\end{aligned}
$$

which is a contradiction. Also, $X^{*}(x)=\frac{d \mu^{a}}{d Q}$ is unique since $U$ is strictly convex. Finally, a monotonicity argument shows that the support of any optimal $\mu_{s}^{*}$ is contained in $\operatorname{argmax} \varphi$.

iii): The dual problem $\inf _{y>0}(v(y)+x y)$ admits a unique minimizer $y(x)$ for all fixed $x>0$. 
a) $x \leq x_{0}$.

$y(x)$ is the unique solution of the equation $-v^{\prime}(y)=x$, i.e. $-E_{P}\left[\frac{d Q}{d P} V^{\prime}\left(y(x) \frac{d Q}{d P}\right]=x\right.$. Setting $X^{*}(x)=-V^{\prime}\left(y(x) \frac{d Q}{d P}\right)=\left(U^{\prime}\right)^{-1}\left(y(x) \frac{d Q}{d P}\right)$, Fenchel equality yields

$$
U\left(X^{*}(x)\right)=V\left(y(x) \frac{d Q}{d P}\right)+y(x) \frac{d Q}{d P} X^{*}(x)
$$

whence $E\left[U\left(X^{*}(x)\right)\right]=v(y(x))+x y(x)$. From $u(x)=\inf _{y>0}\{v(y)+x y\}, X^{*}(x) \in \mathcal{C}(x)$ is the unique optimal payoff.

b) $x>x_{0}$.

The minimizer of the dual problem is constant, $y(x)=y\left(x_{0}\right)=y_{0}$. Setting $X^{*}(x)=X^{*}\left(x_{0}\right)=$ $-V^{\prime}\left(y_{0} \frac{d Q}{d P}\right)=\left(U^{\prime}\right)^{-1}\left(y_{0} \frac{d Q}{d P}\right)$, now $E_{Q}\left[X^{*}(x)\right]=x_{0} \leq x$. An application of Fenchel equality again yields

$$
U\left(X^{*}(x)\right)=U\left(X^{*}\left(x_{0}\right)\right)=V\left(y_{0} \frac{d Q}{d P}\right)+y_{0} \frac{d Q}{d P} X^{*}\left(x_{0}\right)
$$

Taking expectations, $E_{P}\left[U\left(X^{*}(x)\right)\right]=v\left(y_{0}\right)+x_{0} y_{0}$. Then

$$
u(x)=\inf _{y>0}(v(y)+x y)=v\left(y_{0}\right)+x y_{0}=E\left[U\left(X^{*}(x)\right)\right]+y_{0}\left(x-x_{0}\right)
$$

By $i$ ) above, any optimal $\mu_{s}^{*}$ must satisfy $\mu_{s}^{*}(\Omega)=x-x_{0}$.

Corollary 3.4. If $v(y)<+\infty$ for all $y>0$ (in particular if $A E(U)<1$ ), then $y_{0}=0$ and $x_{0}=v_{+}^{\prime}(0)=+\infty$. So the optimal solution is of the form $\mu=X d Q$ for all $x>0$.

Proof. The Inada condition $U^{\prime}(0)=+\infty$ imply that $V^{\prime}(0)=-\infty$, whence:

$$
x_{0}=\lim _{y \downarrow 0}-v^{\prime}(y)=\lim _{y \downarrow 0}-E_{P}\left[\frac{d Q}{d P} V^{\prime}\left(y \frac{d Q}{d P}\right)\right]=+\infty
$$

The thesis follows from Theorem $1.5 \mathrm{iii})$.

Remark 3.5. The Corollary shows that $y_{0}=0$ implies that $x_{0}=+\infty$. However, the reverse implication fails, see Example 4.2.

\section{Examples and Counterexamples}

The examples below explain the role of the singular utility function $\varphi$, and the role of the condition $\varphi=0$ a.s. and Assumption 1.4. The utility function $U$ used is the one defined implicitly by its conjugate $V(y)=e^{1 / y}$ for $y>0$ and $-\infty$ otherwise. Thus, $U(x)=\inf _{y>0}(V(y)+x y)=V(\hat{y})+x \hat{y}$, where $\hat{y}$ is the unique solution to the equation $V^{\prime}(y)=-x$, i.e. $e^{1 / y} / y^{2}=x$. Since this is a trascendental equation, $U$ does not admit a simple expression in terms of elementary functions.

Nevertheless, $U$ satisfies the Inada conditions because $V^{\prime}(0)=-\infty$ and $V^{\prime}(\infty)=0$. Similarly, $U(0)=1$ and $U(\infty)=\infty$ because $V(0)=\infty$ and $V(\infty)=1$. Finally, $U$ has asymptotic elasticity equal to 1 :

$$
\lim _{x \uparrow \infty} \frac{x U^{\prime}(x)}{U(x)}=\lim _{y \downarrow 0}-\frac{V^{\prime}(y) y}{V(y)-y V^{\prime}(y)}=1
$$


and therefore it violates the assumptions of Kramkov and Schachermayer (1999). Since it is also twice-differentiable, de l'Hôpital's rule implies that $U$ is asymptotically risk-neutral, i.e.:

$$
\lim _{x \uparrow \infty}-\frac{x U^{\prime \prime}(x)}{U^{\prime}(x)}=0
$$

Example 4.1 (Infinitely many primal solutions). Consider a bounded double sequence $\left(\omega_{k}\right)_{k \in \mathbb{Z}_{0}} \subseteq \mathbb{R}$, with downward limit $\omega_{-\infty}$ and upward limit $\omega_{+\infty}$, and set $\Omega=\left(\omega_{k}\right)_{k \in \mathbb{Z}_{0}} \cup\left\{\omega_{-\infty}, \omega_{+\infty}\right\}$. Endowed with the Euclidean topology, $\Omega$ is a compact Polish space. Define $P$ by $P\left(\omega_{k}\right)=c_{1}|k|^{-3} e^{-|k|}$ and $P\left(\omega_{-\infty}\right)=P\left(\omega_{+\infty}\right)=0$, and set $\frac{d Q}{d P}\left(\omega_{k}\right)=c_{2} /|k|$ where $c_{1}, c_{2}$ are normalizing constants.

A simple calculation shows that $v(y)=E\left[V\left(y \frac{d Q}{d P}\right)\right]$ is finite iff $y \geq 1 / c_{2}=y_{0}$, and that $v_{+}^{\prime}\left(y_{0}\right)$ is also finite and equal to $-2 c_{1} c_{2} \sum_{n \geq 1} 1 / n^{2}$. In particular, the no-bliss condition $\sup _{X \in \mathcal{C}(x)} E_{P}[U(X)]<$ $\infty$ is satisfied. Moreover, since $\frac{d P}{d Q}$ is finite on $\mathbb{Z}_{0}$, and any $\omega_{k} \in \mathbb{Z}_{0}$ is an isolated point, it follows that $\varphi\left(\omega_{k}\right)=0$ for all $k \in \mathbb{Z}_{0}$, while $\varphi\left(\omega_{-\infty}\right)=\varphi\left(\omega_{+\infty}\right)=y_{0}$. Thus, $\{\varphi>0\}=\left\{\omega_{-\infty}, \omega_{+\infty}\right\}$ is a negligible set and $\varphi=0$ a.s. holds. Assumption 1.4 holds since $\Omega$ is compact.

For $x \leq x_{0}=-v_{+}^{\prime}\left(y_{0}\right)$, the problem admits a classical solution $X^{*}\left(\omega_{k}\right)=\frac{d \mu_{a}^{*}}{d Q}\left(\omega_{k}\right)$, identified by the system:

$$
\begin{aligned}
U^{\prime}\left(X^{*}\left(\omega_{k}\right)\right) & =y \frac{d Q}{d P}\left(\omega_{k}\right) \quad k \in \mathbb{Z}_{0} \\
\sum_{k \in \mathbb{Z}_{0}} X^{*}\left(\omega_{k}\right) Q\left(\omega_{k}\right) & =x
\end{aligned}
$$

When $x>x_{0}$, the above system no longer admits a solution, because the second equality cannot be achieved for any choice of the Lagrange multiplier $y$. The singular utility closes this gap, replacing the previous system by the relaxed system:

$$
\begin{aligned}
U^{\prime}\left(\frac{\mu_{a}^{*}}{d Q}\left(\omega_{k}\right)\right) & =y \frac{d Q}{d P}\left(\omega_{k}\right) \quad k \in \mathbb{Z}_{0} \\
\mu_{s}\left(\omega_{-\infty}\right)+\mu_{s}\left(\omega_{+\infty}\right)+\sum_{k \in \mathbb{Z}_{0}} \frac{d \mu_{a}^{*}}{d Q}\left(\omega_{k}\right) Q\left(\omega_{k}\right) & =x
\end{aligned}
$$

which contains the two additional unknowns $\mu_{s}\left(\omega_{-\infty}\right)$ and $\mu_{s}\left(\omega_{+\infty}\right)$. The solution to the relaxed system is obtained by choosing $y=y_{0}$. The value of $\mu_{s}\left(\omega_{-\infty}\right)+\mu_{s}\left(\omega_{+\infty}\right)$ is thus determined from the second equation, but the two individual values $\mu_{s}\left(\omega_{-\infty}\right)$ and $\mu_{s}\left(\omega_{-\infty}\right)$ remain free. Indeed, since the singular utility term is $\int \varphi d \mu_{s}$, and $\varphi\left(\omega_{-\infty}\right)=\varphi\left(\omega_{+\infty}\right)$, any measure of the form:

$$
\mu^{*}(x)=X^{*}\left(x_{0}\right) d Q+\left(x-x_{0}\right)\left(t \delta_{\omega_{-\infty}}+(1-t) \delta_{\omega_{+\infty}}\right)
$$

for any $t \in[0,1]$ is an optimal solution.

Example $4.2\left(x_{0}=\infty\right.$, but $\left.y_{0}>0\right)$. Consider a bounded sequence $\left(\omega_{n}\right)_{n \geq 1} \subset \mathbb{R}$ decreasing to $\omega_{\infty}$, and define $\Omega$ as $\left(\omega_{n}\right)_{n \geq 1} \cup\left\{\omega_{\infty}\right\}$, endowed with the Euclidean topology, under which it is Polish compact. Define $P$ by $P\left(\omega_{n}\right)=p_{n}=e^{-n} /(e-1)$ and $P\left(\omega_{\infty}\right)=p_{\infty}=0$. The payoff set is defined as $\mathcal{C}(x)=\left\{X \mid E_{Q}[X] \leq x\right\}$, where $Q$ by $\frac{d Q}{d P}\left(\omega_{n}\right)=c_{1} / n$, where $c_{1}>1$ is a normalizing constant, and the value at $\omega_{\infty}$ is irrelevant. As in the previous example, a simple calculation shows that $v(y)=E_{P}[V(y d Q / d P)]$ is finite iff $y>1 / c_{1}:=y_{0}>0$. Thus, the no-bliss condition $\sup _{X \in \mathcal{C}(x)} E_{P}[U(X)]<\infty$ is satisfied, and $\varphi\left(\omega_{n}\right)=0$ for $n \geq 1$ and $\varphi\left(\omega_{\infty}\right)=\frac{1}{c_{1}}=y_{0} . \varphi=0$ a.s. and Assumption 1.4 holds because $\{\varphi>0\}=\left\{\omega_{\infty}\right\}$ is a $P$-negligible set and $\Omega$ is compact. 
In this model, $x_{0}=-v_{+}^{\prime}\left(y_{0}\right)=+\infty$ and therefore the optimal payoff $X^{*}$ is classical for any $x>0$, obtained as the unique solution to the system of equations:

$$
\begin{aligned}
U^{\prime}\left(X^{*}\left(\omega_{n}\right)\right) & =y \frac{d Q}{d P}\left(\omega_{n}\right) \\
\sum_{n \geq 1} X^{*}\left(\omega_{n}\right) q_{n} & =x
\end{aligned}
$$

Example 4.3 (Dropping $\varphi=0$ a.s.). Let $\Omega$ be as in the previous example, but modify $P$ so that $P\left(\omega_{\infty}\right)>0$. More precisely, fix $\delta \in(0,1)$, and define $P$ by $p_{n}=P\left(\omega_{n}\right)=(1-\delta) e^{-n} /(e-1)$ and $p_{\infty}=P\left(\omega_{\infty}\right)=\delta$. Likewise, define $Q$ by $\frac{d Q}{d P}\left(\omega_{n}\right)=1 / n$ and $Q\left(\omega_{\infty}\right)=\left(1-\sum_{n \geq 1} Q\left(\omega_{n}\right)\right)>0$. Now, $v(y)$ is finite iff $y>1$, so $y_{0}=1$. Since the continuous function:

$$
g_{k}\left(\omega_{n}\right)=\left\{\begin{array}{cc}
\frac{1}{k} & \text { if } n \leq k \\
1+\frac{1}{k} & \text { if }+\infty \geq n>k
\end{array}\right.
$$

is in $\operatorname{Dom}\left(J_{V}\right)$ for all $k \geq 1, \varphi\left(\omega_{n}\right)=0$ for all $1 \leq n<+\infty$, while $\varphi\left(\omega_{\infty}\right)=1$. For $\omega_{\infty}$, the following holds:

$$
W\left(\omega_{\infty}, x\right)=\max _{z \leq x}\left\{U(z)+(x-z) \varphi\left(\omega_{\infty}\right) \frac{d Q}{d P}\left(\omega_{\infty}\right)\right\}
$$

and consider the derivative

$$
U^{\prime}(x)-\varphi\left(\omega_{\infty}\right) \frac{d Q}{d P}\left(\omega_{\infty}\right)
$$

If $x>x^{*}=\left(U^{\prime}\right)^{-1}\left(\varphi\left(\omega_{\infty}\right) \frac{d Q}{d P}\left(\omega_{\infty}\right)\right), W$ attains its maximum at $x^{*}$, so that

$$
W\left(\omega_{\infty}, x\right)=\left\{\begin{array}{cl}
U(x) & \text { if } x \leq x^{*} \\
U\left(x^{*}\right)+\left(x-x^{*}\right) \varphi\left(\omega_{\infty}\right) \frac{d Q}{d P}\left(\omega_{\infty}\right) & \text { if } x>x^{*}
\end{array}\right.
$$

Example 4.4 (Necessity of Assumption 1.4). The setup is the same of Example 4.1 above, only remove the points $\omega_{-\infty}, \omega_{+\infty}$. The resulting $\Omega$ is no longer compact, but still Polish with the Euclidean topology. As $\frac{d P}{d Q}$ is now finite everywhere and the topology is discrete, $\varphi$ is identically null. However, the value function $u$ is the same of Example 4.2, so in particular for $x>x_{0}$

$$
u(x)=\sup _{\mathcal{C}(x)} E[U(X)]=E\left[U\left(X^{*}\left(x_{0}\right)\right)\right]+y_{0}\left(x-x_{0}\right)
$$

and the extra contribution cannot be given by a singular measure $\mu_{s}^{*}$ - only by a pure finitely additive $\mu_{p}^{*}$ with $\inf _{f \in \operatorname{Dom}\left(J_{V}\right)} \mu_{p}^{*}(f)=y_{0}\left(x-x_{0}\right)>0$. The maximizing sequences in $\mathcal{C}(x)$ for the value $u(x)$ are the same of Example 4.2, but this time the sequences have a weak star cluster point in $\mathcal{D}(x) \backslash \mathcal{M}_{+}$.

\section{References}

Aliprantis, C. D. and Border, K. C. (2006), Infinite dimensional analysis, third edn, Springer, Berlin. A hitchhiker's guide.

Ambrosio, L., Gigli, N. and Savaré, G. (2008), Gradient flows in metric spaces and in the space of probability measures, Lectures in Mathematics ETH Zürich, second edn, Birkhäuser Verlag, Basel. 
Ball, J. M. and Murat, F. (1989), 'Remarks on Chacon's biting lemma', Proc. Amer. Math. Soc. $\mathbf{1 0 7}(3), 655-663$.

Benninga, S. and Mayshar, J. (2000), 'Heterogeneity and option pricing', Review of Derivatives Research 4(1), 7-27.

Borwein, J. M. and Lewis, A. S. (2006), Convex analysis and nonlinear optimization, CMS Books in Mathematics/Ouvrages de Mathématiques de la SMC, 3, second edn, Springer, New York. Theory and examples.

Bouchitté, G. and Valadier, M. (1988), 'Integral representation of convex functionals on a space of measures', J. Funct. Anal. 80(2), 398-420.

Brezis, H. (1983), Analyse fonctionnelle, Collection Mathématiques Appliquées pour la Maîtrise. [Collection of Applied Mathematics for the Master's Degree], Masson, Paris. Théorie et applications. [Theory and applications].

Buttazzo, G. (1989), Semicontinuity, relaxation and integral representation in the calculus of variations, Vol. 207 of Pitman Research Notes in Mathematics Series, Longman Scientific \& Technical, Harlow.

Cvitanic, J. and Malamud, S. (2008), 'Asset Prices, Funds' Size and Portfolio Weights in Equilibrium with Heterogeneous and Long-Lived Funds'. Working paper, ETH Zurich and Caltech.

Dunford, N. and Schwartz, J. T. (1988), Linear operators. Part I, Wiley Classics Library, John Wiley \& Sons Inc., New York. General theory, With the assistance of William G. Bade and Robert G. Bartle, Reprint of the 1958 original, A Wiley-Interscience Publication.

Kramkov, D. and Schachermayer, W. (1999), 'The asymptotic elasticity of utility functions and optimal investment in incomplete markets', Ann. Appl. Probab. 9(3), 904-950.

Kramkov, D. and Schachermayer, W. (2003), 'Necessary and sufficient conditions in the problem of optimal investment in incomplete markets', Ann. Appl. Probab. 13(4), 1504-1516.

Rockafellar, R. T. (1974), Conjugate duality and optimization, Society for Industrial and Applied Mathematics, Philadelphia, Pa. Lectures given at the Johns Hopkins University, Baltimore, Md., June, 1973, Conference Board of the Mathematical Sciences Regional Conference Series in Applied Mathematics, No. 16.

Schachermayer, W. (2002), Optimal investment in incomplete financial markets, in 'Mathematical finance-Bachelier Congress, 2000 (Paris)', Springer Finance, Springer, Berlin, pp. 427-462.

Yosida, K. and Hewitt, E. (1952), 'Finitely additive measures', Trans. Amer. Math. Soc. 72, 46-66. 